\title{
CARACTERIZACIÓN DE LA OPERACIÓN DE SECADO DE NANCITE (Byrsonima crassifolia)
}

\section{CHARACTERIZATION OF THE DRYING OPERATION OF NANCITE (Byrsonima crassifolia)}

\author{
Daniela Magaly Ruiz Saldívar ${ }^{1}$ \\ Sandra Lorena Blandón Navarro ${ }^{2 *}$
}

(Recibido/received: 29-octubre-2021; aceptado/accepted: 19-noviembre-2021)

RESUMEN: El nancite (Byrsonima crassifolia) es una fruta tropical perecedera, cuyo periodo de cosecha se da al inicio del invierno (mes de mayo) en los países de América Central. Los frutos se suelen consumir crudos, en refrescos, jaleas, jarabes o mermeladas, como saborizante para helados y como materia prima para productos de elaboración artesanal. No obstante, es necesario continuar promoviendo más alternativas de valor agregado para dicho producto. En ese sentido, estudios previos sugieren que la pulpa de nancite fresca aporta $66 \mathrm{kcal}$ por cada 100 gramos y presenta un contenido de materia seca entre 20 y $25 \%$, lo que puede ser aprovechado en su agroindustrialización como producto deshidratado. Por lo tanto, en esta investigación se estudió el proceso de secado de la pulpa de nancite en términos del impacto de la temperatura en las tasas de secado y en el color del producto final. A través del análisis proximal de la parte comestible del nancite (pulpa) se determinó un contenido de humedad de $78.80 \pm 0.32 \%$ (b.h) y en base seca se determinó un contenido de carbohidratos de $78.67 \pm 0.53$ y extracto etéreo de $16.60 \pm 0.27 \%$. Los experimentos de secado de este material se realizaron en secador de túnel usando aire seco con una velocidad de $2 \mathrm{~m} / \mathrm{s}$ a temperaturas de $60^{\circ} \mathrm{C}, 70^{\circ} \mathrm{C}$ y $80^{\circ} \mathrm{C}$. Los resultados sugieren que el contenido de humedad y el tiempo de secado disminuyen conforme se incrementa la temperatura de operación, sin embargo, a $60^{\circ} \mathrm{C}$ el producto seco presenta el menor pardeamiento, por lo que esta temperatura de secado podría recomendarse en procesos en los que se requiera una pulpa seca de color amarillo pálido.

PALABRAS CLAVE: nanche; deshidratación; composición química; fruta tropical

ABSTRACT: Nancite (Byrsonima crassifolia) is a perishable tropical fruit and its harvest period occurs at the beginning of winter (month of May) in the countries of Central America. The fruits are usually consumed raw, in soft drinks, jellies, syrups or jams, as a flavoring for ice cream and as a raw material for artisanal food. However, it is necessary to continue promoting more valueadded alternatives for this product. In this sense, previous studies suggest that fresh nancite pulp

1 Profesora, Universidad Nacional Autónoma de Nicaragua, POLISAL UNAN-MANAGUA. Departamento de Bioanálisis clínico y Microbiología. Managua, Nicaragua

${ }^{2}$ Profesora titular, Universidad Nacional de Ingeniería, Sede Regional del Norte, Estelí, Nicaragua. *Autor para correspondencia, email: sandra.blandon@norte.uni.edu.ni 
contributes $66 \mathrm{kcal}$ per 100 grams and has a dry matter content between 20 and $25 \%$, which can be used in its agro-industrialization as a dehydrated product. Therefore, in this research, the nancite pulp drying process was studied in terms of the impact of temperature on the drying rates and on the color of the final product. Through the proximal analysis of the edible part of the nancite (pulp) a moisture content of $78.80 \pm 0.32 \%$ (on a wet basis) was determined and on a dry basis a carbohydrate content of $78.67 \pm 0.53$ and an ether extract of $16.60 \pm 0.27 \%$ were determined. The drying experiments of this material were carried out in a tunnel dryer using dry air with a speed of $2 \mathrm{~m} / \mathrm{s}$ at temperatures of $60^{\circ} \mathrm{C}, 70^{\circ} \mathrm{C}$ and $80^{\circ} \mathrm{C}$. The results suggest that moisture content and drying time decrease as the operating temperature increases, however, at $60^{\circ} \mathrm{C}$ the dry product presents the least browning, so this drying temperature could be recommended in processes in which that a pale yellow dry pulp is required.

KEYWORDS: nanche; dehydration; chemical composition; tropical fruit.

\section{INTRODUCCIÓN}

El fruto del nancite (Byrsonima crassifolia), es de consumo popular en países de la América tropical y subtropical como México, Colombia, Venezuela y Brasil. Pertenece a la familia Mapighiaceae, la cual comprende 63 géneros y 1110 especies aproximadamente (Benezar \& Pessoni, 2006).

El fruto es una pequeña drupa globosa, de 1 a $2 \mathrm{~cm}$ de diámetro, epicarpio o cáscara delgada, color verde (inmaduro) y amarillo (maduro); la parte comestible está constituida por el mesocarpio de color amarillo, suave y pastoso, con cerca de $0.5 \mathrm{~cm}$ de espesor y olor y sabor característicos. El endocarpio ovalado, leñoso, conteniendo una, dos o tres semillas viables (Otzoy Rosales y Alvarado Güinac, 2003).

Bayuelo, Lozano y Ochoa (2006) en estudio de la caracterización morfológica de Byrsonima crassifolia (I.) kunth nativa de Churumuco, Michoacán, México, reportaron que las características morfológicas del árbol y fruto, así como las características químicas de la pulpa (acidez del fruto, sólidos solubles totales y proteína), son las que resultan determinantes en la variabilidad del nancite.

La pulpa de nancite representa aproximadamente entre el $79 \%$ (Gusmão, de Almeida Vieira y Meira da Fonseca Júnior, 2006) y $89.5 \%$ (Maldonado Peralta et al., 2020) del peso total de la fruta fresca. Además, contiene alrededor de $82.8 \%$ de humedad y es buena fuente de nutricional con $14.4 \mathrm{~g}$ de carbohidratos, $1.3 \mathrm{~g}$ de lípidos, $0.90 \mathrm{~g}$ de proteínas, para un aporte de $66 \mathrm{kcal}$ por cada 100 gramos de pulpa fresca (IBGE, 1999). El fruto se comercializa en su mayoría sin ninguna preparación, pero los que se comercialización procesados se incluyen en mermeladas, almíbar, helados, granizados y fermentados, como bebida alcohólica (Otzoy Rosales y Alvarado Güinac, 2003). Por lo tanto, es necesario seguir impulsando alternativas de mayor valor agregado para esta fruta tropical, como aquellos procesos que incluyen operaciones de acondicionamiento y secado de la pulpa. 
El secado es una técnica de conservación de alimentos cuyo objetivo principal es la disminución de la actividad del agua de los mismos. Al mismo tiempo, el secado influye en las propiedades físicas de los materiales. La superficie se seca y cambia su capacidad para absorber o reflejar la luz. Además, la contracción y la deformación de la superficie también afectan la reflexión de la luz. La evaporación del agua da como resultado la concentración de solubles, también colorantes y la contracción del tejido conduce a una estructura compacta (Lewicki \& Duszczyk, 1998).

Esto acarrea a la necesidad de estudiar los factores que determinan la calidad del producto terminado y que pueden ayudar a establecer los parámetros del procedimiento de secado. De esta manera, se puede contribuir a optimizar el proceso en términos de economía y energía. Por lo tanto, se realizó la presente investigación con la finalidad de identificar la temperatura adecuada de secado de la pulpa de nancite, el tiempo necesario para llevar a cabo el proceso y el impacto en el color del producto seco.

\section{MATERIALES Y MÉTODOS}

Los análisis de la composición química de la pulpa de nancite fueron realizados en el Laboratorio de Biotecnología de la Universidad Nacional Autónoma de Nicaragua (UNAN-Managua). Los experimentos de secado se llevaron a cabo en el Laboratorio de Procesos de la Facultad de Ingeniería Química de la Universidad Nacional de Ingeniería (FIQ UNI, Managua, Nicaragua).

Este estudio es experimental, ya que se basa en experimentos de secado, en los cuales se evaluó el efecto de la temperatura $\left(60,70\right.$ y $\left.80^{\circ} \mathrm{C}\right)$ sobre el tiempo de secado de la pulpa de nancite, además del efecto sobre el color del producto final. Guevara Alban, Verdesoto Arguello y Castro Molina (2020) plantean que en los estudios experimentales se somete a un objeto o grupo de individuos en determinadas condiciones, estímulos o tratamiento (variable independiente), para observar los efectos o reacciones que se producen (variable dependiente). En este caso, el objeto de estudio (muestra) es la pulpa de nancite.

\section{Muestra}

Los frutos de Nancite, peso de $10 \mathrm{~kg}$, fueron comprados en el mercado de Mayoreo, de la ciudad de Managua en agosto del 2019. Posteriormente, pasaron por una operación de selección, para asegurar que la muestra se encontrara en una etapa ideal de maduración (pulpa amarillenta) y apta para consumo fresco (pulpa de textura firme y suave).

\section{Métodos analíticos}

Para el análisis de la composición química se determinó el contenido de humedad con el método AOAC 925.45, materia seca AOAC 920.151, cenizas AOAC 940.26, extracto etéreo AOAC 920.39. Para calcular el porcentaje de proteínas se aplicó el método AOAC 2001.11, Kjeldahl que se basa en digestión de la muestra de pulpa de nancite seca, seguido de destilación y titulación del destilado y, finalmente, la cuantificación de la composición proteica multiplicándose el contenido de nitrógeno por el factor 6.25 (A.O.A.C., 2005). Para el cálculo del contenido de carbohidratos se realizó por diferencia, con la siguiente ecuación: 
Contenido de carbohidratos totales (\%) = Materia seca - (lípidos + proteínas + cenizas) \%

Todos estos análisis se realizaron por duplicado.

Los sólidos solubles (en grados Brix) de la pulpa de nancite, obtenida de los frutos maduros, se midió con un refractómetro portátil AMTAST (escala de 0 a 32 grados Brix). Para ello, se colocaron dos gotas de la muestra en la superficie del prisma y se procedió a realizar la lectura.

El valor de $\mathrm{pH}$ fue obtenido introduciendo en la muestra de pulpa de nancite el electrodo del medidor de pH Fisherbrand Accumet AP85. Tanto la medición del pH como de los grados Brix se realizaron por quintuplicado.

Acondicionamiento de muestra

Las principales operaciones unitarias a las que fue sometido el fruto de nancite en la etapa de acondicionamiento de la materia prima, se describen a seguir.

\section{a. Limpieza}

La eliminación de impurezas es una operación que debe realizarse en todas las materias primas. Por ello, los frutos de nancite fueron sometidos a lavado y sanitización mediante la inmersión en solución de hipoclorito de sodio (50 ppm).

\section{b. Selección}

Se seleccionaron frutos que presentaron firmeza, con apariencia (forma y color ideales frutos maduros), ausencia de lesiones, cortes y podredumbre, oscurecimiento y manchas negras en la cutícula.

\section{c. Despulpado}

Se extrajo la pulpa de manera manual, separando la semilla. A seguir, la pulpa se almacenó en bolsas de polietileno a $-18^{\circ} \mathrm{C}$, hasta su utilización en los experimentos.

\section{Experimentos de secado}

El secado es una operación que tiende a reducir el contenido de humedad de un producto y que se lleva a cabo, por lo general, mediante aire caliente.

El equipo utilizado fue un secador de túnel convectivo, que consta de cuatro secciones: sección de suministro de gas y deshumidificación, sección de calentamiento, cámara de secado y equipo de análisis (Picado, Alfaro y Gamero, 2021).

Para cada una de las corridas experimentales la muestra (pulpa de nancite obtenida de los frutos maduros) se colocó en el porta-muestra del equipo de modo que ocupara todo su volumen el cual es $16.87 \mathrm{~cm}^{3}$. Se registró el peso inicial de la muestra con la balanza analítica. El porta-muestra con la pulpa, se colocó luego sobre la balanza en la sección del secador de túnel de modo que el producto estuviera expuesto a las condiciones requeridas por el experimento. Esquema del equipo está representado en el estudio realizado por Picado et al. (2021). 
La pérdida de peso durante el secado fue determinada mediante su medición en la balanza analítica y se realizó cada 10 minutos hasta que el peso de las muestras fuese constante.

Las condiciones de secado evaluadas en los experimentos fueron temperaturas de 60, 70 y 80 ${ }^{\circ} \mathrm{C}$ y a velocidad del aire constante de $2 \mathrm{~m} / \mathrm{s}$ para los tres tratamientos, con dos repeticiones para cada uno.

El efecto de la temperatura sobre el color de las muestras se evaluó mediante observación, comparándolas unas con otras.

Procesamiento y Análisis de los resultados

Los datos de la investigación se procesaron en el Software estadístico InfoStat ® versión 2018 (Di Rienzo et al., 2018) para realizar la comparación de medias por el test de Duncan (95 \% de confianza).

\section{RESULTADOS Y DISCUSIÓN}

En la caracterización del fruto de nancite verde y maduro (amarillo) se obtuvieron diferencias estadísticamente significativas con una $p<0.05$ (test de Duncan) para el ancho, largo, y diámetro de la semilla (mm), como mostrado en la Tabla 1.

Tabla 1. Caracterización física del fruto de Nancite (Byrsonima crassifolia)

\begin{tabular}{llll}
\hline Fruto & Ancho $(\mathrm{mm})$ & Largo $(\mathrm{mm})$ & Diámetro de semilla $(\mathrm{mm})$ \\
\hline Nancite Verde & $14.33 \pm 0.52 \mathrm{a}$ & $13.33 \pm 0.52 \mathrm{a}$ & $7.00 \pm 0.00 \mathrm{a}$ \\
\hline Nancite & $16.67 \pm 1.37 \mathrm{~b}$ & $14.83 \pm 0.75 \mathrm{~b}$ & $8.00 \pm 0.00 \mathrm{~b}$ \\
Amarillo & & & \\
\hline
\end{tabular}

Los datos son expresados como la media y la desviación estándar. Medias en la misma columna con letras minúsculas diferentes, difieren significativamente entre sí.

Los valores presentados en la tabla 1 para el ancho y largo del nancite son menores a los reportados por Maldonado Peralta et al. (2020) con diámetros para el fruto de 1.7 a $2 \mathrm{~cm}$. No obstante, los resultados de este estudio coinciden en que los frutos evaluados tienen forma de oblato; es decir, son más anchos que largos. Maldonado Peralta et al. (2020) mencionan que este es un parámetro importante de calidad, ya que incide en el tipo de empaque a utilizar y a la vez sobre la preferencia del consumidor. Moreno Gutiérrez (2000) también menciona que el fruto del nancite presenta colores amarillentos a tonos ligeramente naranja. Además, contiene abundante pulpa agridulce que rodea al endocarpio. El exocarpio es delgado, de color amarillo, verde o rojizo cuando el fruto está maduro; el mesocarpio (la parte comestible) es de consistencia pastosa, de color amarillo y de unos $5 \mathrm{~mm}$ de espesor, el endocarpio es redondeado, rígido y reticulado. La semilla se encuentra encerrada en el endocarpio que es duro y leñoso; de forma ovoide, gruesa o ligeramente comprimida de 4 a $4.5 \mathrm{~mm}$ de diámetro aproximadamente. Esto último difiere de lo determinado en la presente investigación. Variaciones en las caracteristicas 
de los frutos están asociadas a las variaciones climáticas, condiciones de manejo, genotipos (Bayuelo-Jiménez, Lozano Rico y Ochoa, 2006).

En la figura 1 se presenta el fruto de nancite en diferentes etapas de desarrollo y maduración. Es posible observar la diferencia en el diámetro entre los frutos verdes y los maduros. El crecimiento del fruto del nancite ocurre en 56 días, período en que llega a su máximo diámetro ecuatorial ( 18 mm) (Medina-Torres, Salazar-García, Valdivia-Bernal y Martínez-Moreno, 2012), de manera que los frutos verdes no han alcanzado su desarrollo completo, lo que puede explicar el por qué los frutos verdes presentan diámetro menor.

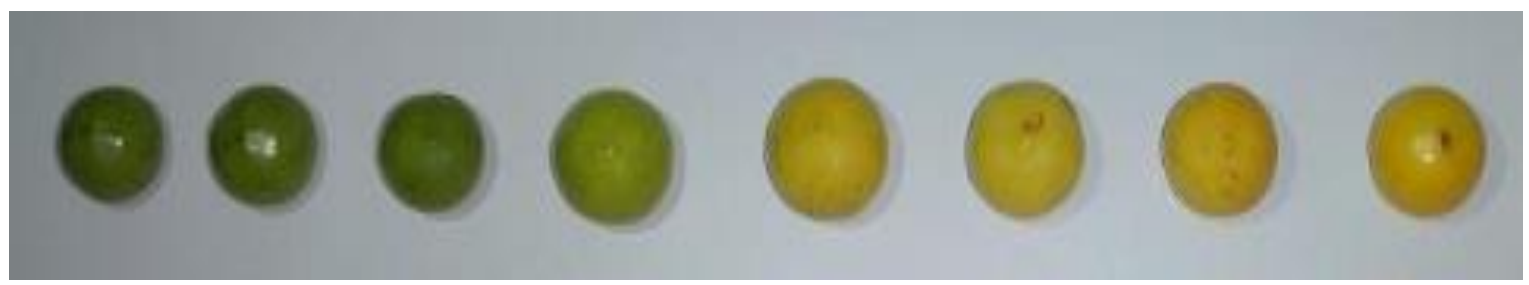

Figura 1. Fruto del nancite en los estadíos de verde a amarillo (madurez organoléptica)

La pulpa de nancite obtenida de los frutos maduros, presentó pH de $3.03 \pm 0.02$ y contenido de sólidos solubles de $15.33 \pm 0.52$ grados Brix. El valor de pH está dentro del rango reportado por Medina-Torres et al. (2015) de 2.65 a 3.94 para nancites dulces cuyo contenido de sólidos solubles varía de 10.96 a 14.07 grados Brix. No obstante, existen genotipos de nancite que pueden presentar contenido mayor de sólidos solubles (Medina-Torres et al., 2015). En este sentido, Bayuelo-Jiménez et al. (2006) determinaron que el nancite dulce presenta contenido de sólidos solubles en el rango de 9.7 a $18 \%$.

Del análisis proximal de la parte comestible del fruto del Nancite maduro (la pulpa), se obtuvieron los siguientes resultados, en base húmeda : Humedad $78.80 \pm 0.32 \%$, materia seca $21.20 \pm 0.32$ $\%$, de la cual las cenizas corresponden $0.74 \pm 0.03$, extracto etéreo $3.52 \pm 0.01$, proteinas $0.26 \pm$ 0.00 y carbohidratos totales de $16.57 \pm 0.20 \%$. En cuanto al contenido de extracto etéreo es menor al reportado por Vilarins Silva (2016) de $7.6 \%$ en base húmeda para muestras colectadas en el municipio de Amarante del estado Maranhão (Brasil). Esta diferencia puede deberse al origen geográfico, respuesta a factores tanto bióticos y abióticos y variabilidad genética de la planta.

En base seca, la pulpa de nancite posee extracto etéreo $16.60 \pm 0.27$, proteínas $1.24 \pm 0.02$, cenizas $3.49 \pm 0.17$ y carbohidratos $78.67 \pm 0.53 \%$. Esta composición sugiere que la pulpa seca podría aportar hasta $460 \mathrm{kcal}$ por cada 100 gramos, valor que es relevante y que debe ser considerado en la preparación de formulaciones a base de este alimento.

Con respecto a la masa de humedad eliminada por gramo de muestra (pulpa de nancites maduros) en el proceso de secado a $60^{\circ} \mathrm{C}$ en la figura 2 se observa que a partir de los 270 minutos cesa la pérdida de humedad, alcanzando la muestra seca un peso constante. 


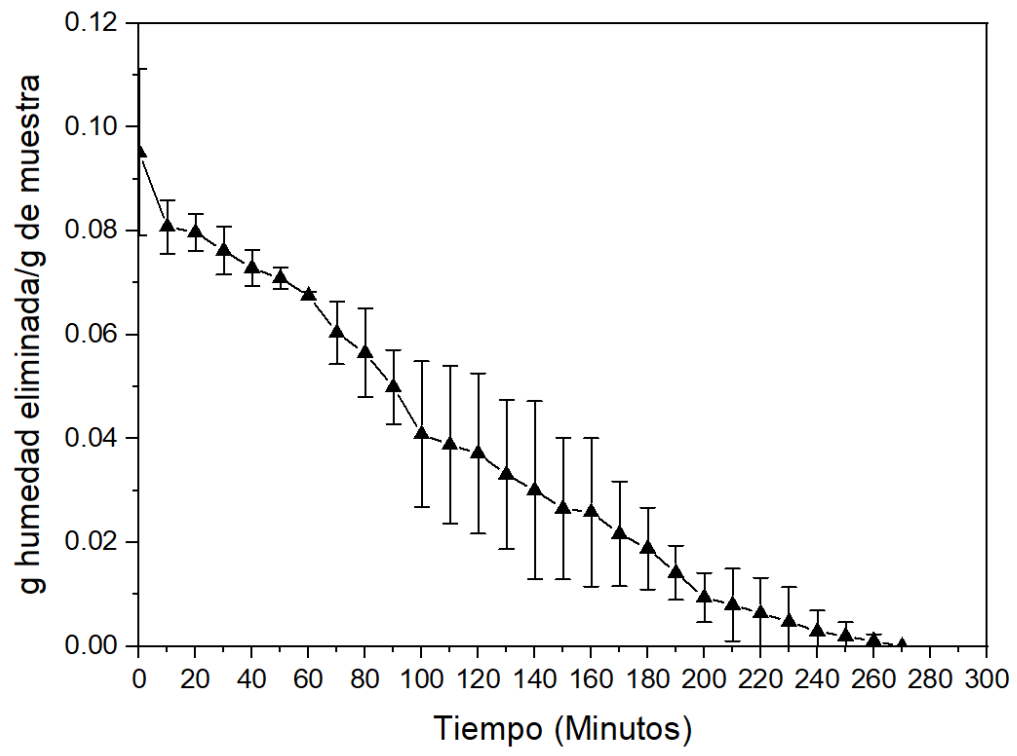

Figura 2. Variación de la humedad de la pulpa de nancite (g) en el proceso de secado a $60{ }^{\circ} \mathrm{C}$ En la Figura 3 la masa de humedad eliminada por gramo de muestra en el proceso de secado a $70{ }^{\circ} \mathrm{C}$ va disminuyendo con respecto al tiempo, cesando la pérdida de humedad a los 210 minutos, siendo este tiempo inferior que el registrado para la temperatura de $60^{\circ} \mathrm{C}$.

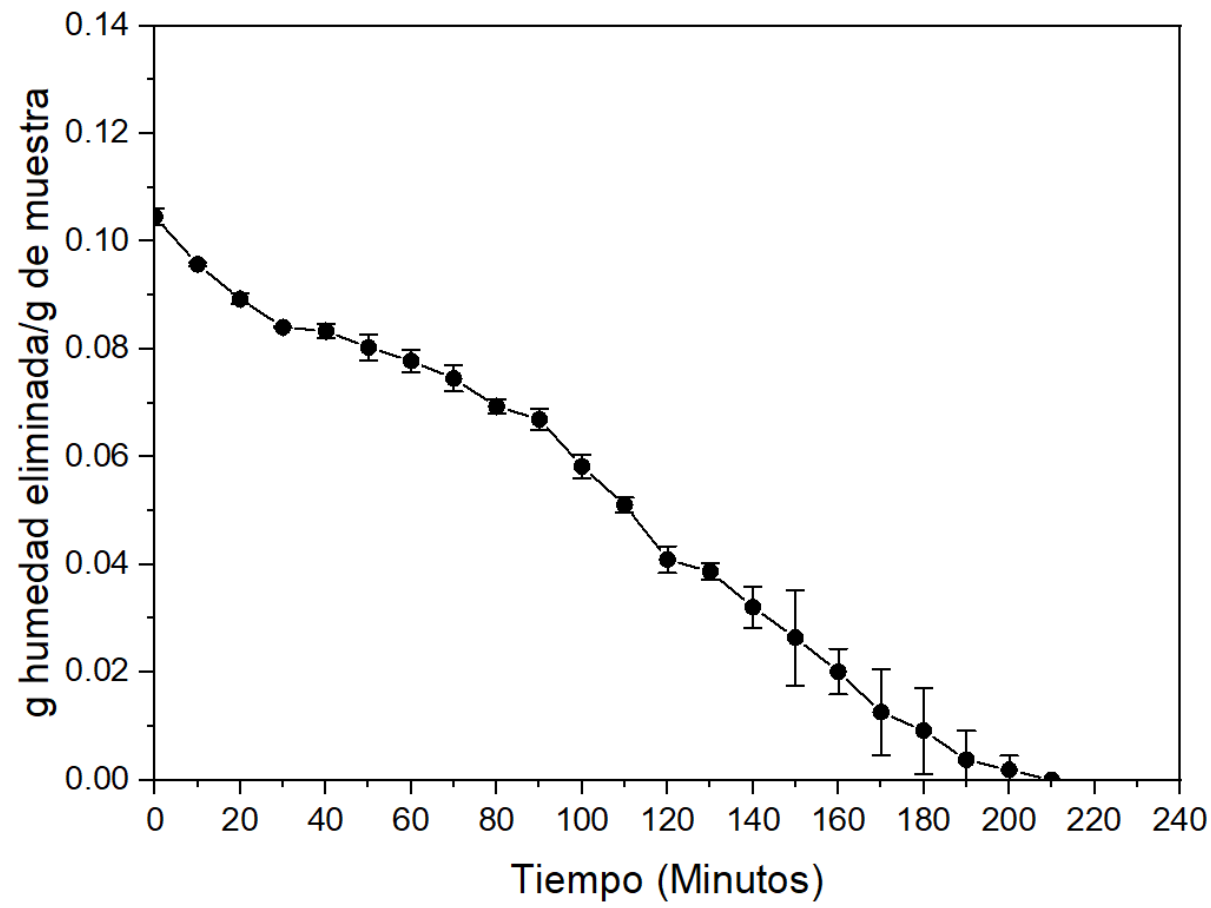

Figura 3. Variación de la humedad de la pulpa de nancite (g) en el proceso de secado a $70^{\circ} \mathrm{C}$ 
La variación de la humedad de la pulpa de nancite (g) por gramo de muestra en el proceso de secado a $80^{\circ} \mathrm{C}$ se muestra en la Figura 4 . A esta temperatura, la pérdida de humedad cesa a los 160 minutos. A partir de este tiempo, la muestra alcanza un peso constante, lo que evidencia que la muestra está seca.

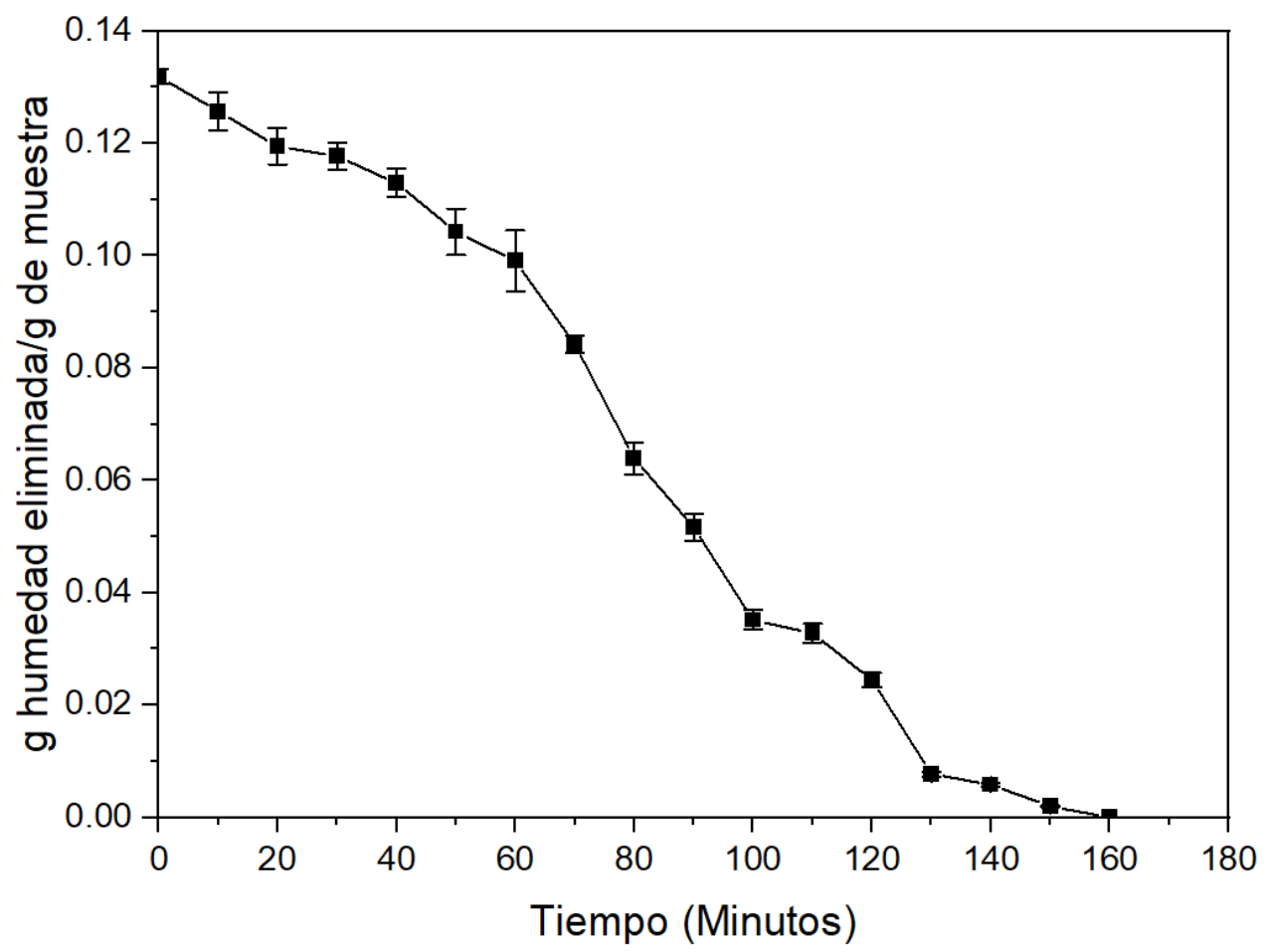

Figura 4. Variación del peso de la pulpa de nancite $(\mathrm{g})$ en el proceso de secado a $80^{\circ} \mathrm{C}$

Los tiempos de secado determinados en este estudio difieren de los reportados por Modesto Antunes et al. (2017), debido a las diferencias en el acondicionamiento de la pulpa, previo al secado. Modesto Antunes et al. (2017) adicionaron agentes emulsionantes a la pulpa de nancite y posteriormente sometieron a agitación la mezcla hasta formar una capa espumosa. De este modo, los autores reportaron tiempos de secado de 150 minutos (2.5 horas), 162 minutos (2.7 horas) y 102 minutos ( 1.5 horas) a 60,70 y $80^{\circ} \mathrm{C}$, respectivamente.

En los experimentos desarrollados pudo determinarse que a $80^{\circ} \mathrm{C}$ se consigue un secado más rápido, sin embargo en el secado a $60^{\circ} \mathrm{C}$ se observa menor pardeamiento en la pulpa seca. El pardeamiento no enzimático tiene varias causas, como la reacción de los azúcares reductores con los aminoácidos, la caramelización del azúcar, la descomposición de la vitamina $\mathrm{C}$ y la destrucción de los pigmentos (Damasceno, Fernandes, Magalhães, \& Brito, 2008). El aumento de la temperatura de secado favorece estas reacciones. En la figura 5 es posible observar la diferencia en el color de las muestras secadas en las diferentes temperaturas.

El cambio de color entre la pulpa fresca y las muestras secas a las tres temperaturas estudiadas podría ser debido al pardeamiento no enzimático, concentración de la materia seca y cambios en la estructura, como sugerido por Lewicki y Duszczyk (1998). Así, los cambios de color de los 
materiales secos pueden deberse también a la eliminación de agua, la sustitución del agua por aire y la deformación de la superficie (contracción).
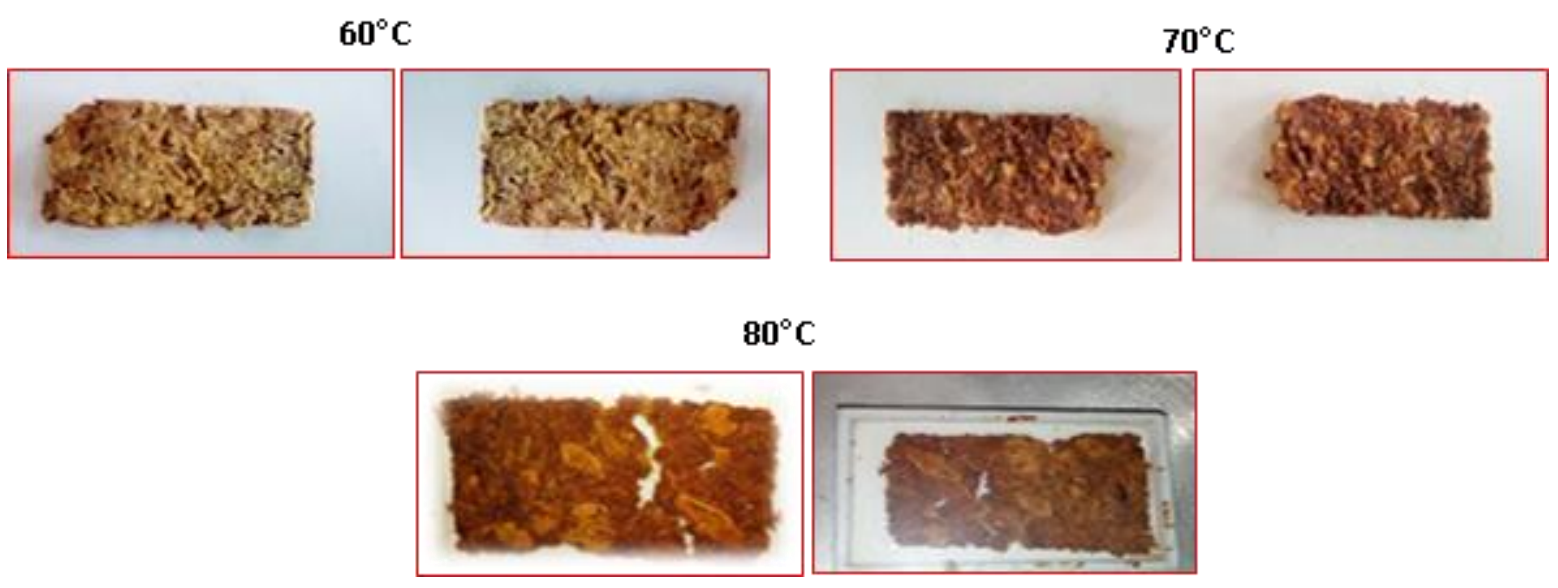

Figura 5. Variaciones del color de la pulpa de nancite a diferentes temperaturas de secado

\section{CONCLUSIONES}

De las principales caracteristicas macroscópicas del fruto de nancite verde y maduro (amarillo) se obtuvieron diferencias estadísticamente significativas con una $p<0.05$ (test de Duncan) para el ancho, largo, y grosor de la pulpa $(\mathrm{mm})$. En los análisis proximales los constituyentes químicos de la parte comestible del fruto del Nancite, en base húmeda : Humedad $78.80 \pm 0.32 \%$, materia seca con $21.20 \pm 0.32 \%$, cenizas $0.74 \pm 0.03$, extracto etéreo para un $3.52 \pm 0.01$, proteinas con $0.26 \pm 0.00$ y carbohidratos totales de $16.57 \pm 0.20 \%$. En base seca, la pulpa de nancite presenta extracto etéreo $16.60 \pm 0.27$, proteinas $1.24 \pm 0.02$, cenizas $3.49 \pm 0.17$ y carbohidratos $78.67 \pm 0.53$, respectivamente.

Con respecto al comportamiento a escala de laboratorio de la curva de secado se determinó que la velocidad de este proceso depende de la temperatura utilizada. Así, las muestras secadas a 60 y $70{ }^{\circ} \mathrm{C}$ requieren de 270 minutos y 210 minutos, respectivamente, mientras que a $80{ }^{\circ} \mathrm{C}$ se requieren solamente 160 minutos. No obstante, la temperatura de $80^{\circ} \mathrm{C}$ es la que más impacta en el color del producto, debido al aumento del pardeamiento no enzimático. De este modo, cuando se desee que el producto seco presente menos pardeamiento se sugerirá el secado a 60 ${ }^{\circ} \mathrm{C}$.

\section{BIBLIOGRAFÍA}

A.O.A.C. (2005). Official Methods of Analysis of AOAC International (18th ed.). Arlington, Virginia: AOAC International.

Bayuelo-Jiménez, J. S., Lozano Rico, J. C., Ochoa, I. E. (2006). Caracterización morfológica de byrsonima crassifolia (I.) kunth nativa de Churumuco, Michoacán, México. Revista Fitotecnia Mexicana, 29(2), 31-36. 
Benezar, R. M. C., \& Pessoni, L. A. (2006). Biologia floral e sistema reprodutivo de Byrsonima coccolobifolia (Kunth) em uma savana amazônica. Acta Amazonica, 36(2), 159-168. https://doi.org/10.1590/S0044-59672006000200005

Damasceno, L. F., Fernandes, F. A. N., Magalhães, M. M. A., Brito, E. S. (2008). Evaluation and optimization of non enzymatic browning of "cajuina" during thermal treatment. Brazilian Journal of Chemical Engineering, 25(2), 313-320. https://doi.org/10.1590/S010466322008000200010

Di Rienzo, J. A., Casanoves, F., Balzarini, M. G., Gonzalez, L., Tablada, M., \& Robledo, C. W. (2018). Infostat versión 2018. Córdoba, Argentina: Universidad Nacional de Córdoba. Retrieved from http://www.infostat.com.ar

Guevara Alban, G. P., Verdesoto Arguello, A. E., Castro Molina, N. E. (2020). Metodologías de investigación educativa (descriptivas, experimentales, participativas, y de investigaciónacción). RECIMUNDO, 4(3), 163-173. https://doi.org/10.26820/recimundo/4.(3).julio.2020.163-173

Gusmão, E., de Almeida Vieira, F., Meira da Fonseca Júnior, É. (2006). Biometria de frutos e endocarpos de murici (Byrsonima verbascifolia Rich. ex A. Juss.). CERNE, 12(1), 84-91.

IBGE. (1999). Estudo nacional de despesa familiar: Tabelas de composição de alimentos. (5ta ed.). Rio de Janeiro: Instituto Brasileiro de Geografia e Estatística.

Lewicki, P. P., Duszczyk, E. (1998). Color change of selected vegetables during convective air drying. International Journal of Food Properties, 1(3), 263-273. https://doi.org/10.1080/10942919809524582

Maldonado Peralta, M. de los Á., Santillán, P. S., García, A. R. R., Lagarda, J. L. V., Luzardo, M. B. B., Gutiérrez, L. A. (2020). Caracterización y evaluación de frutos de 'nanche' (Byrsonima crassifolia L.). Revista Mexicana de Ciencias Agrícolas, 11(1), 151-160. https://doi.org/10.29312/remexca.v11i1.1950

Medina-Torres, R., Juárez-López, P., Salazar-García, S., López-Guzmán, G. G. Ibarra-Sánchez, L. S., Arrieta-Ramos, B. G., Martínez-Moreno, E. (2015). Evaluación de calidad en frutos de 41 genotipos de nanche (Byrsonima crassifolia L. HBK) de Nayarit, México. Revista Mexicana de Ciencias Agrícolas, 6(2), 253-264.

Medina-Torres, R., Salazar-García, S., Valdivia-Bernal, R., Martínez-Moreno, E. (2012). Fenología de la floración y ciclos reproductivos del nanche [Byrsonima crassifolia (L.) HBK] en Nayarit. Universidad y Ciencia, 28(3), 259-269.

Modesto Antunes, A., Rosa Evangelista, Z., Morais Santos, M., Alessandro Devilla, I., Fernandes Lisboa, C., Gomes de Oliveira, D. (2017). Mathematical modeling of the pulp drying curves murici (Byrsonima crassifolia): The foam layer drying. African Journal of Agricultural Research, 12(18), 1547-1551. https://doi.org/10.5897/ajar2016.11996

Moreno Gutiérrez, M. N. (2000). El nance Byrsonima crassifolia (L.) HBK como recurso natural antimicrobiano en enfermedades gastrointestinales y respiratorias (No. Sirsi) i9789685149112). Tuxtla Gutiérrez, Chiapas, México: Universidad de Ciencias y Artes del estado de Chiapas.

Otzoy Rosales, M., Alvarado Güinac, D. (2003). Caracterización Agromorfológica de 74 materiales de Nance (Byrsonima spp.) colectados en la región Sur-Occidental de Guatemala. Mazatenango, Guatemala: Programa Universitario de Investigación en Recursos Naturales y Ambiente (PUIRNA).

Picado, A., Alfaro, S., Gamero, R. (2021). Drying kinetics and thermodynamic analysis of tomato 
in a tunnel dryer. Nexo Revista Científica, 34(03), 31-45. https://doi.org/10.5377/nexo.v34i03.11859

Vilarins Silva, R. (2016). Avaliação da estabilidade termo-oxidativa do óleo de murici (Byrsonima crassifolia L. Kunt) obtido apartir de hidrólise enzimática assistida por ultrasom. Universidade Federal do Maranhão.

\section{SEMBLANZA DE LOS AUTORES}

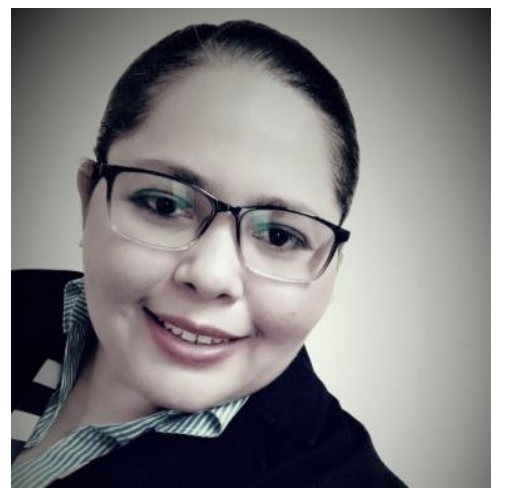

Daniela Magaly Ruiz Saldívar: Es Máster en procesamiento de alimentos, Universidad Nacional de Ingeniería (UNI), Nicaragua 2019 y graduada de Licenciada en Bioanálisis Clínico, Universidad Nacional Autónoma de Nicaragua (2010). Actualmente es docente de la UNAN-Managua a nivel de grado, en la carrera de Bioanálisis clínico y Microbiología (POLISAL). Posee gran experiencia en el diagnóstico microbiológico desempeñándose por durante 10 años en el Hospital Solidaridad, Nicaragua. Educación online Optimización del uso de Antimicrobianos/ Gestión de la resistencia a los antimicrobianos. University of Dundee. Además, posee experiencia en el área de Microbiología de los alimentos, con énfasis en el diagnóstico microbiológico y en la elaboración de productos utilizando microorganismos benéficos.

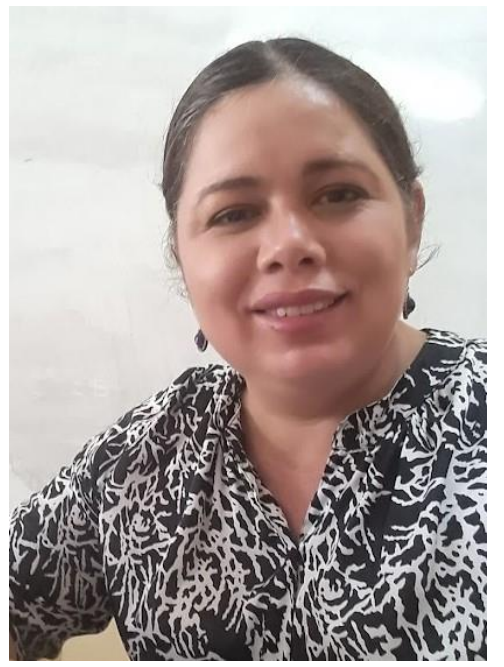

Sandra Lorena Blandón Navarro: Es Doctora en Ciencias de Ingeniería de Alimentos, Universidad de Sao Paulo (USP), Brasil (2016), con maestría en Procesamiento de Alimentos, Universidad Nacional de Ingeniería (UNI), Nicaragua (2009) y graduada de Ingeniería Química en la misma universidad (2003). Actualmente es Profesora titular de la UNI a nivel de grado y posgrado, en la carrera de Ingeniería agroindustrial y en la maestría en Procesamiento de Alimentos de la Facultad de Ingeniería Química (FIQ-UNI). Posee diplomados de Modelo Educativo Institucional (IPN-México), Educación Online (UOLUNI, Managua, Nicaragua) y Estrategias de enseñanza y aprendizaje aplicadas a los estudios de alimentos (ISEKI FOOD4 PROJECT, Atenas, Grecia). Además, posee experiencia en el área de Ciencia y Tecnología de Alimentos, con énfasis en desarrollo de productos, aprovechamiento de residuos de la agroindustria e ingeniería de separaciones. 